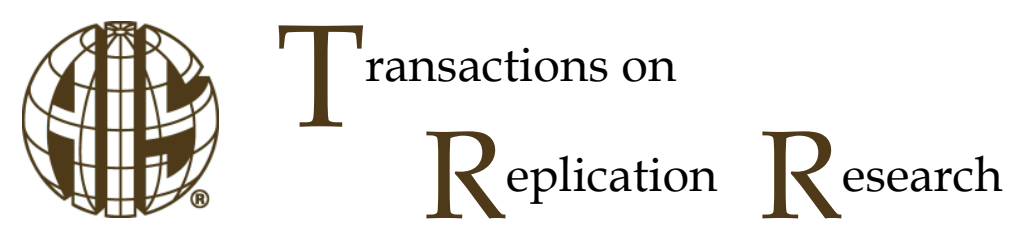

\title{
Open Materials Discourse: Enhancement of Recall within Technology-Mediated Teams Through the Use of Online Visual Artifacts
}

\author{
K. Asli Basoglu \\ Lerner College of Business \& Economics \\ University of Delaware \\ asli@udel.edu
}

\author{
Mark A. Fuller \\ Isenberg School of Management \\ University of Massachusetts \\ dean@isenberg.umass.edu
}

\author{
Joseph S. Valacich \\ Eller College of Management \\ University of Arizona \\ valacich@email.arizona.edu
}

Abstract:

This paper provides the materials used to conduct the Basoglu, Fuller, and Valacich (2012) study of individuals' recall of information within technology-mediated teams (i.e., virtual teams). In the Basoglu et al. (2012) study, three input factors-visual artifacts (i.e., a computer-generated image of each team member), team size, and work interruptionswere manipulated to assess their influence on a person's ability to recall important characteristics of their virtual team members. In the current paper, we provide step-by-step instructions for the experimental design, procedures, consent form, and the questionnaire administered by Basoglu et al. (2012). Our objectives are to provide researchers with the necessary materials to replicate the Basoglu et al. (2012) study and use its methodological techniques to guide their own research.

Keywords: Open materials, Interruptions, Virtual teams, Laboratory experiments, Virtual artifacts 


\section{Introduction}

The objective of this paper is to provide the materials used to conduct Basoglu, Fuller, and Valacich (2012) study of individuals' recall of information within technology-mediated teams (i.e., virtual teams). In the Basoglu et al. (2012) study, three input factors-visual artifacts (i.e., a computer-generated image of each team member), team size, and work interruptions-were manipulated to assess their influence on a person's ability to recall important characteristics of their virtual team members. Results showed that providing participants with images of their virtual team members significantly increased participants' recall performance. However, a high-urgency interruption that required participants to divert from their experimental tasks significantly deteriorated recall performance, and this effect occurred regardless of team size or whether the team member images were provided. In the current paper, we provide step-by-step instructions for the experimental design, procedures, consent form, and the questionnaire administered by Basoglu et al. (2012). Our objective is to provide researchers with the necessary materials to replicate the Basoglu et al. (2012) study and use its methodological techniques to guide their own research.

\section{Research Design}

The Basoglu et al. (2012) study used a $2 \times 2 \times 2$ factorial experiment to test their four hypotheses - Figure 1 depicts their research model. The three between-participant factors were visual artifact (with vs. without the images of the virtual team members), team size (large vs. small), and interruption urgency (low vs. high). In other words, each participant was part of either a large (8 member) or small (4 member) team, with or without the visual artifact, while being exposed to either a high- or low-urgency interruption. As described below, this interruption took the form of an email that either required urgent attention or where the participant could respond the next day. For participants that were assigned to a large team, they received more cues or team member profile information as compared to participants assigned to small teams. The ordering of the team member cues or information was counterbalanced to eliminate concerns regarding sequencing effects. To assess the impact of the interruption urgency manipulation, the study implemented a control group with no interruption for each of the visual artifact treatments.

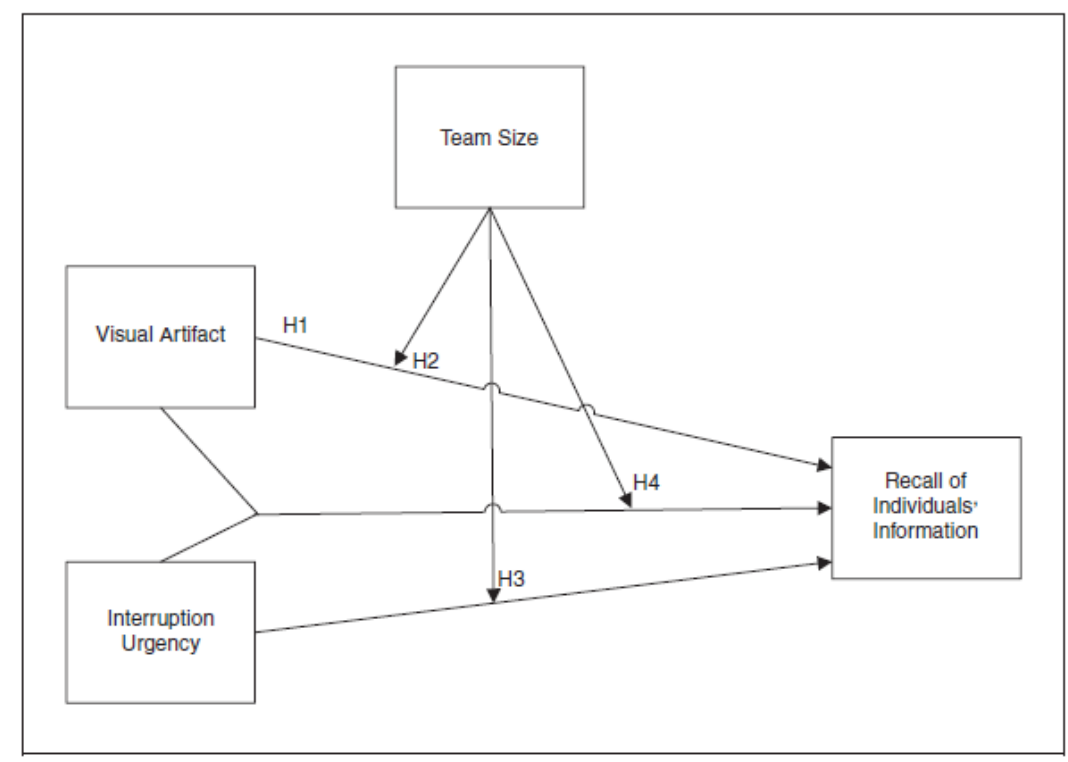

Figure 1. Research Model from Basoglu et al. (2012)

\subsection{Experimental Task}

The experiment is designed to take place in a computer lab. For the Basoglu et al. (2012) study, computer stations were set up prior to each session where browsers/screens were closed and informed consent forms were placed on the keyboards. The lab proctor greeted participants as they walked in, and asked them to 
select a station of their choice. Participants were provided the following instructions, which were displayed on a large computer screen in the front of the room:

"Please do not to use the computers until instructed. In the meantime, please silence your phones, and put them away. If you finish the experiment early, please stay seated until the session is over. Please note that this is a voluntary study and you are permitted to terminate your session at any point in time. The information collected will be kept confidential."

Next, the lab proctor asked the participants to carefully read the consent form and sign it. Once the forms were collected, the lab proctor sent the survey link to all the computer stations through a lab management software to start the experiment

\subsubsection{Informed Consent Form}

The approximate time for the experiment is 15-25 minutes. You must follow the instructions to be rewarded. Moreover, you are expected to use your judgment to decide how to respond to distractions during the study.

\section{Confidentiality}

The information collected in this study will be kept confidential. Data will be secured and will be made available only to persons conducting the study unless you give permission in writing to do otherwise.

\section{Approval}

Please note that this study has been reviewed by the (insert your institution here) Institutional Review Board (IRB) for human subject participation. If you have questions about your rights as a participant please contact the (insert your institution here) IRB at (insert the telephone number and email for the IRB contact at your institution here).

\section{Compensation}

There will be a random drawing for ten gift certificates ( $\$ 5-\$ 10$ each) as a reward to participants who demonstrate effective decision making in this experiment.

\section{Participation}

Your participation in this study is voluntary; you may decline to participate. If you decide to participate, you may withdraw from the study voluntarily at any time without any penalty.

If you have any questions about the study or the procedures, or if you experience adverse effects as a result of participating in this study, you may contact XXX, at the XXX College of Business, Dept. of XXX; phone number and email (insert the appropriate contact information here).

\section{Consent}

I have read and understand the above information. I agree to participate in this study.

Investigator's Signature:

\subsubsection{Debriefing Email}

Once all the experimental sessions were completed, the participants were sent the following debriefing email. "Dear participant, thank you for your participation in our study! Your participation is greatly appreciated. The goal of our research was to understand the impact of interruptions on memory recall. If you would like to receive a copy of the final report of this study (or a summary of the findings) when it is completed, please feel free to contact the researcher" (researcher's name, email address, and phone numbers should be provided here). 


\section{Experimental Conditions}

As described, the Basoglu et al. (2012) study employed a $2 \times 2 \times 2$ factorial design. Below are the summary descriptions of each of the experimental conditions.

\begin{tabular}{|c|c|c|c|c|}
\hline $\begin{array}{c}\text { Cond. } \\
\quad \#\end{array}$ & $\begin{array}{c}\text { Team } \\
\text { Size }\end{array}$ & $\begin{array}{l}\text { Visual } \\
\text { Artifact }\end{array}$ & $\begin{array}{l}\text { Interruption } \\
\text { Urgency }\end{array}$ & Explanation \\
\hline 1 & Small & No & High & $\begin{array}{l}\text { Four group members are introduced with no visual artifact (Figure } \\
\text { 8). All participants are interrupted with a high-urgency interruption } \\
\text { (Figure 6). }\end{array}$ \\
\hline 2 & Small & Yes & High & $\begin{array}{l}\text { Four group members are introduced with visual artifacts (Figure } \\
\text { 3). All participants are interrupted with a high-urgency interruption } \\
\text { (Figure 6). }\end{array}$ \\
\hline 3 & Small & No & Low & $\begin{array}{l}\text { Four group members are introduced with no visual artifact (Figure } \\
\text { 8). All participants are interrupted with a low-urgency interruption } \\
\text { (Figure 4). }\end{array}$ \\
\hline 4 & Small & Yes & Low & $\begin{array}{l}\text { Four group members are introduced with visual artifacts (Figure } \\
\text { 3). All participants are interrupted with a low-urgency interruption } \\
\text { (Figure 4). }\end{array}$ \\
\hline 5 & Large & Yes & High & $\begin{array}{l}\text { Eight group members are introduced with visual artifacts (Figure } \\
\text { 5). All participants are interrupted with a high-urgency interruption } \\
\text { (Figure 6). }\end{array}$ \\
\hline 6 & Large & No & High & $\begin{array}{l}\text { Eight group members are introduced with no visual artifact. All } \\
\text { participants are interrupted with a high-urgency interruption } \\
\text { (Figure 6). }\end{array}$ \\
\hline 7 & Large & No & Low & $\begin{array}{l}\text { Eight group members are introduced with no visual artifact. All } \\
\text { participants are interrupted with a low-urgency interruption (Figure } \\
\text { 4). }\end{array}$ \\
\hline 8 & Large & Yes & Low & $\begin{array}{l}\text { Eight group members are introduced with visual artifacts (Figure } \\
\text { 5). All participants are interrupted with a high-urgency interruption } \\
\text { (Figure 6). }\end{array}$ \\
\hline 9 & Small & No & - & Control Group \\
\hline 10 & Small & Yes & - & Control Group \\
\hline 11 & Large & No & - & Control Group \\
\hline 12 & Large & Yes & - & Control Group \\
\hline
\end{tabular}

In the remainder of this paper, we provide the materials used to create the experimental conditions that are described in Table 1. Note that we do not provide every possible combination of treatments (i.e., the 12 conditions in Table 1) but with the information provided, researchers can create each of these conditions when conducting the experiment. Depicted in this paper are three conditions. The first condition is the small team size, with visual artifact, and low interruption urgency manipulation - condition \#4 in Table 1. This experimental condition entails background information about the experiment, which is shown in Figure 2; the introduction of four team members with visual artifacts, which is shown in Figure 3; and the interruption by a low-urgency email, which is shown in Figure 4. After these aspects of the experiment, participants completed the post-experiment questionnaire. This questionnaire included eight items that measured participants' recall of their team members' information, demographics, manipulation checks, participants' perceptions about the attractiveness of the visual artifacts, interest in the interruption task, and follow-up questions about the details of the low-urgency interruption email. The details of the post-experiment questionnaire for condition \#4 are provided in Tables 2 and 3. 
Please read the following information very carefully.

One of the technologies that are now being used to support teamwork in organizations are virtual worlds. Virtual worlds are computer-based simulated environments where users interact with one another through avatars (computer generated images that users choose to represent themselves), rather than by their actual photos.

For this research, we're asking you to assume that you work at company that is using virtual worlds for this purpose. Please read the descriptions associated with the following potential teammates. Make sure to read the descriptions carefully and try to remember as much detail about members as possible, because you will be asked questions about these potential teammates later and you will not be able to return to these descriptions once you have finished reading them.

When you are done familiarizing yourself with your potential teammates, you will be asked to continue onto the next phase of the task.

Figure 2. Background Information about the Experiment Provided to All Participants

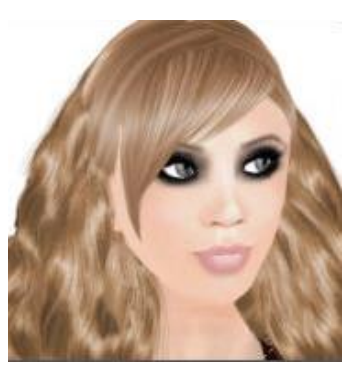

$\mathrm{Hi} ! ! !$

- My name is Jordan.

- I live in Washington DC and I am an airline pilot.

- I have been playing violin since I was six.

- Last month I hiked in the Appalachians for 3 months.

- I also play lacrosse for a co-ed recreational team.

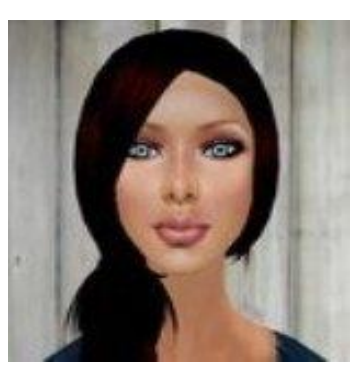

Hello everyone!

- My name is Jamie.

- I am 25 years old.

- I work in the New York Stock Exchange Market.

- I spent a year in Africa with the Peace Corps.

- I also enjoy parachuting.

Figure 3: Team Member Information for Small Team with Visual Artifacts (Each Displayed on a Single Webpage 


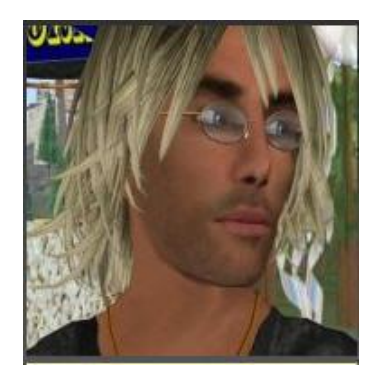

Hi there!

- My name is Alex.

- I am an engineer and I build wind turbines and solar energy systems.

- I recently designed and built a solar collector for a home water-heating system.

- I am also an amateur photographer and painter.

- Two years ago, I won the best amateur photographer award.

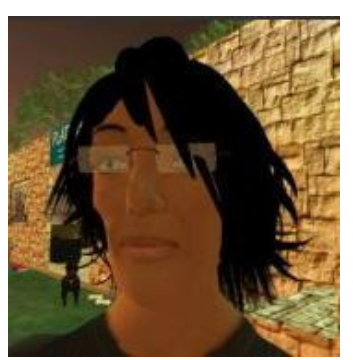

Hey!

- My name is Chris.

- I am a consultant working in aircraft design for NASA.

- I have an engineering degree from University of Massachusetts.

- I also teach engineering at the University of Massachusetts.

- $\quad$ Last year, I took my class to NASA for a field trip.

Figure 4 Cont'd: Team Member Information for Small Team with Visual Artifacts (Each Displayed on a Single Webpage) 
Your boss has just sent the following email.

At some point, you'll need to read the email carefully. Since the email implies you are NOT going to be meeting with your boss until much later to discuss the contents of this message, this email will be saved and you'll be able to access if after you are done with the virtual teamwork task. You can also read it now if you choose.

After you've finished with the virtual teamwork task, you'll be able to access this email again, and answer some preparation questions we've created to help you be prepared for your meeting.

When you are finished with this page, just click next to return to the virtual teamwork task.

From: Your Boss

Sent: Now

To: You

Subject: Virus Outbreak

$\mathrm{Hi}$

I just saw this come through on our email security list. Would you please look it over, and let's talk about it tomorrow.

"A Wifi virus outbreak? Researchers say its' possible"

If criminals were to target unsecured wireless routers, they could create an attack that could piggyback across thousands of Wi-Fi networks in areas like Chicago or New York, according to researchers at Indiana University.

The researchers estimate that a Wi-Fi attack could take over 20,000 wireless routers in New York within a two-week period, with most of the infections occuring within the first day.

"The issue is that most of these routers are installed out of the box very insecurely," said Steven Myers, an assistant professor at Indiana University who published the paper in November, along with researchers from the Institute for Scientfic Interchange in Torino, Italy. The researchers theorize the attack would work by guessing administrative passwords and then instructing the routers to install new worm-like firmware that would in turn cause the infected router to attack other devices in its range.

Because there are so many closely connected Wi-Fi networks in most urban areas, the attack could hop from router to router for many miles in some cities.

The team used what is known as the Suseptible Infected Removed (SIR) model to track the growth of such an attack. The methodology is typically used to estimat the scale of events like influenza outbreaks, but it has also been used to predice comptuer virus infections.

Although the researchers did not develop the attack code that would be used to carry out this type of infection, they believe it would be possible to write code that guessed default passwords by first entering the default administrative passwords that shipped with the routers, and then trying a list of 1 million commonly used passwords, one after the other. They said $36 \%$ of passwords could be guessed using this technique.

Even some routers that use encryption could be cracked, if they use the popular WEP (Wired Equivalent Privacy) algorithm, which security experts have been able to crack for years. Routers that are encrypted using the more secure WPS (Wi-Fi Protected Access) standard are considered impossible to inflect.

We'd now like you to return to our task related to virtual work. Please answer the following questions related to your potential team members.

Figure 5. Low-Urgency Interruption Email 
Table 2. Items for Recall of Team Members' Information - Condition \#4

Please read the description in quotes and select the team member it belongs to.

This person "spent a year in Africa with the Peace Corps".

Who is the person in the description?

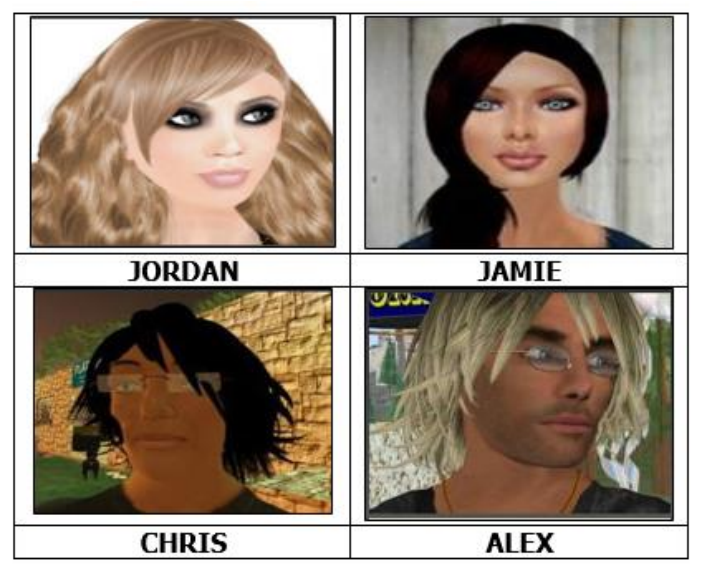

o Jordan o Jamie o Chris o Alex

Q1: This person "plays lacrosse for a co-ed recreational team".

Q2: This person is "an engineer and builds wind turbines and solar energy systems".

Q3: This person "teaches engineering at University of Massachusetts and also works for NASA".

Q4: This person "an airline pilot and hiked in the Appalachians last summer for 3 months".

Q5: This person is an "amateur photographer and painter".

Q6: This person "spent a year in Africa with the Peace Corps"

Q7: This person "works in the New York Stock Exchange Market".

Q8: This person "likes parachuting".

Notes: (1) the above figure was provided for each of the eight items, with the text of the particular item included above the images of the team members; (2) these items appeared on the post-experiment questionnaire, along with items shown in Table 3 below; (3) incorrect answers are recorded as zero and correct answers are recorded as one; the sum of the correct answers obtained determines the participant's recall score. 
Table 3. Remaining Items on the Post-Experiment Questionnaire for Condition \#4

Manipulation Checks (7-point Likert scale with endpoints "Strongly Agree/Disagree").

Q1: Trying to remember all the descriptions about team members was overwhelming.

Q2: I felt like there was a lot of information about the team members.

Q3: The "Virus Outbreak" questions made me forget some of team members' descriptions.

Attractiveness of the Visual Artifact (7-point Likert scale with endpoints "Strongly Agree/Disagree").

Q1: Please rate the overall attractiveness of the team members' pictures.

Q2: Please rate the attractiveness of the following team member's picture: (note: this item provided for each team member picture)

Interest in Interruption Task (7-point Likert scale with endpoints "Strongly Agree/ Disagree").

Q1: I found the "Virus Outbreak" article interesting.

Q2: I enjoyed reading the "Virus Outbreak" article.

Demographics

Please indicate your age.

Please indicate your gender (male/female).

Follow-up Questions about the Interruption Email

Q1: How would the attack work (select one):

Overpowering routers with requests

Sending commands to turn off routers

Guessing administrative passwords and then sending instructions to routers

Bypassing the routers

Q2: Routers that are using New passwords are particularly susceptible (select one):

- Reverse

- Default Non-numeric

Q3: SIR stands for Security Information Requirement

Suspicious Internet Request

Susceptible Infected Removed

Standard Information Reuse

Q4: WEP stands for ___ (select one):

Wireless Equipment Protocol

Wired Equivalent Privacy

Wireless Exclusionary Provision

Wound Evolutionary Propagation

Q5: What percentage of routers could be compromised using the technique in the article (select one): $15 \%$

$28 \%$

$36 \%$

$45 \%$

$60 \%$

Q6: Most infections would occur in The first hour

_ The first day

The first week The first two weeks

Q7: What university were the researchers at (select one): Wisconsin

Indiana

Berkeley

Nebraska (select one): 
The second condition presented in this paper is the large team size, with visual artifact, and high interruption urgency manipulation - condition \#5 in Table 1. This experimental condition entails background information about the experiment, which is shown in Figure 2; the introduction of eight team members with visual artifacts, which is shown in Figure 6; and the interruption by a high-urgency email, which is shown in Figure 7. In this high-urgency condition, the follow-up questions about the interruption email immediately follow the presentation of the email, and these questions are displayed in Table 4. Subsequent is the post-experiment questionnaire, and the items that comprise this questionnaire for condition \#5 are shown in Tables 5 and 6.

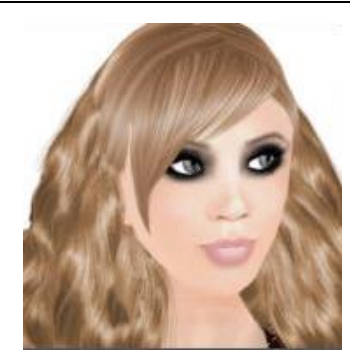

$\mathrm{Hi} ! !$

- My name is Jordan.

- I live in Washington DC and I am an airline pilot.

- I have been playing violin since I was six.

- Last month I hiked in the Appalachians for 3 months.

- I also play lacrosse for a co-ed recretaional team.

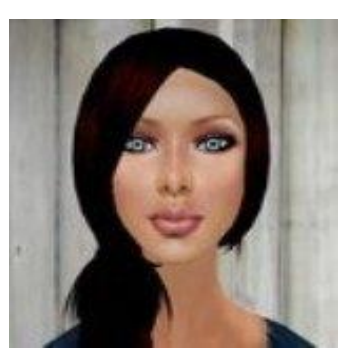

Hello everyone!

- My name is Jamie.

- I am 25 years old.

- I work in the New York Stock Exchange Market.

- I spent a year in Africa with the Peace Corps.

- I also enjoy parachuting.

Figure 5. Team Member Information for Large Team with Visual Artifacts (Each Displayed on a Single Webpage) 


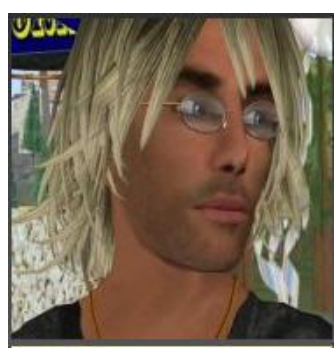

Hi there!

- My name is Alex.

- I am an engineer and I build wind turbines and solar energy systems.

- I recently designed and built a solar collector for a home water-heating system.

- I am also an amateur photographer and painter.

- $\quad$ Two years ago, I won the best amateur photographer award.

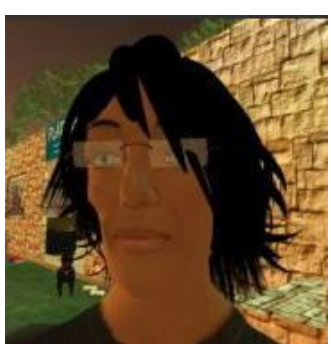

Hey!

- My name is Chris.

- I am a consultant working in aircraft design for NASA.

- I have an engineering degree from University of Massachusetts.

- I also teach engineering at the University of Massachusetts.

- $\quad$ Last year, I took my class to NASA for a field trip.

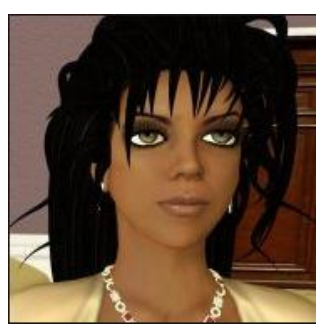

Hi everyone!

- My name is Lee.

- I am a senior at Northwestern University.

- I am good at economics.

- I am the editor of the economics magazine in Northwestern.

- I won the "Most Promising Junior Economist" award last year.

Figure 5 Cont'd. Team Member Information for Large Team with Visual Artifacts (Each Displayed on a Single Webpage) 


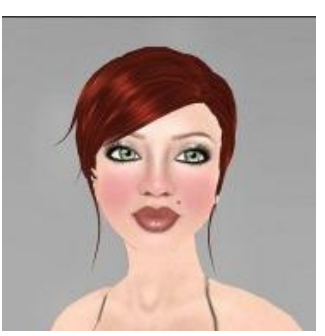

Hello everybody!

- My name is Pat.

- I am a junior at University of Idaho.

- My grandparents are German and they taught me German and Polish.

- I like archeology and I go to Greece every summer for field execution.

- I also enjoy amateur astronomy.

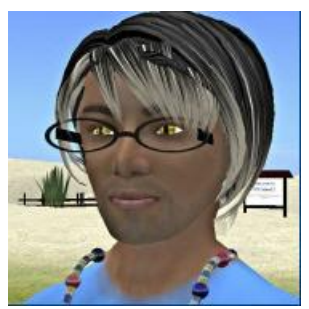

Hi there!

- $\quad$ My name is Blair.

- I am from Chile and I am a make-up artist in Beverly Hills, California.

- I drive a hybrid car to work, recycle paper, cardboard boxes, and cans.

- My favorite hobby is to try different coffee flavors.

- I am traveling to Brazil next summer to attend a special course on coffee tasting.

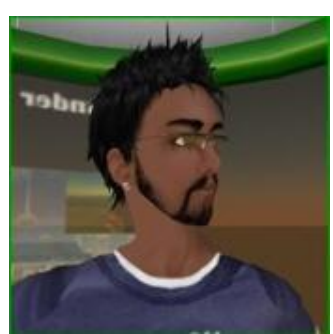

Hey!

- My name is Casey.

- I am a professional actor and I played in multiple shows in Broadway.

- I am originally from New Zealand and went to school in London, UK.

- I also own my own restaurant in New York.

- I enjoy playing Wii with my son.

Figure 5 Cont'd. Team Member Information for Large Team with Visual Artifacts (Each Displayed on a Single Webpage) 
Your boss has just sent the following email.

Please read the email carefully. Since the email implies that you will be meeting your boss shortly and discuss the contents of the email, we've created a set of preparation questions to help you prepare for the meeting. After you finish reading the email, click next to answer the preparation questions.

From: Your Boss

Sent: Now

To: You

Subject: Virus Outbreak

$\mathrm{Hi}$

I just saw this come through on our email security list. Would you please look it over, and let's talk about it in an hour or so.

"A Wifi virus outbreak? Researchers say its' possible"

If criminals were to target unsecured wireless routers, they could create an attack that could piggyback across thousands of Wi-Fi networks in areas like Chicago or New York, according to researchers at Indiana University.

The researchers estimate that a Wi-Fi attack could take over 20,000 wireless routers in New York within a two-week period, with most of the infections occuring within the first day.

"The issue is that most of these routers are installed out of the box very insecurely," said Steven Myers, an assistant professor at Indiana University who published the paper in November, along with researchers from the Institute for Scientfic Interchange in Torino, Italy. The researchers theorize the attack would work by guessing administrative passwords and then instructing the routers to install new worm-like firmware that would in turn cause the infected router to attack other devices in its range.

Because there are so many closely connected Wi-Fi networks in most urban areas, the attack could hop from router to router for many miles in some cities.

The team used what is known as the Suseptible Infected Removed (SIR) model to track the growth of such an attack. The methodology is typically used to estimat the scale of events like influenza outbreaks, but it has also been used to predice comptuer virus infections.

Although the researchers did not develop the attack code that would be used to carry out this type of infection, they believe it would be possible to write code that guessed default passwords by first entering the default administrative passwords that shipped with the routers, and then trying a list of 1 million commonly used passwords, one after the other. They said $36 \%$ of passwords could be guessed using this technique.

Even some routers that use encryption could be cracked, if they use the popular WEP (Wired Equivalent Privacy) algorithm, which security experts have been able to crack for years. Routers that are encrypted using the more secure WPS (Wi-Fi Protected Access) standard are considered impossible to inflect.

We'd now like you to return to our task related to virtual work. Please answer the following questions related to your potential team members.

Figure 6. High-Urgency Interruption Email 
Table 4. Follow-Up Questions about the Interruption Email for Condition \#5*

Q1: How would the attack work (select one):

Overpowering routers with requests

Sending commands to turn off routers

Guessing administrative passwords and then sending instructions to routers

Bypassing the routers

Q2: Routers that are using New

Reverse

Default

Non-numeric

Q3: SIR stands for

Security Information Requirement

_ Suspicious Internet Request Susceptible Infected Removed Standard Information Reuse

Q4: WEP stands for __ (select one):

Wireless Equipment Protocol

Wired Equivalent Privacy

Wireless Exclusionary Provision

Wound Evolutionary Propagation

Q5: What percentage of routers could be compromised using the technique in the article (select one): $15 \%$

$28 \%$

$36 \%$

$45 \%$

$60 \%$

Q6: Most infections would occur in The first hour

The first day

- The first week

The first two weeks

Q7: What university were the researchers at (select one):

Wisconsin

Indiana

Berkeley

Nebraska

${ }^{*}=$ these same follow-up questions come immediately after the email for all high-urgency conditions. 
Table 5. Items for Recall of Team Members' Information - Condition \#5

Please read the description in quotes and select the team member it belongs to.

This person "plays lacrosse for a co-ed recreational team".

Who is the person in the description?

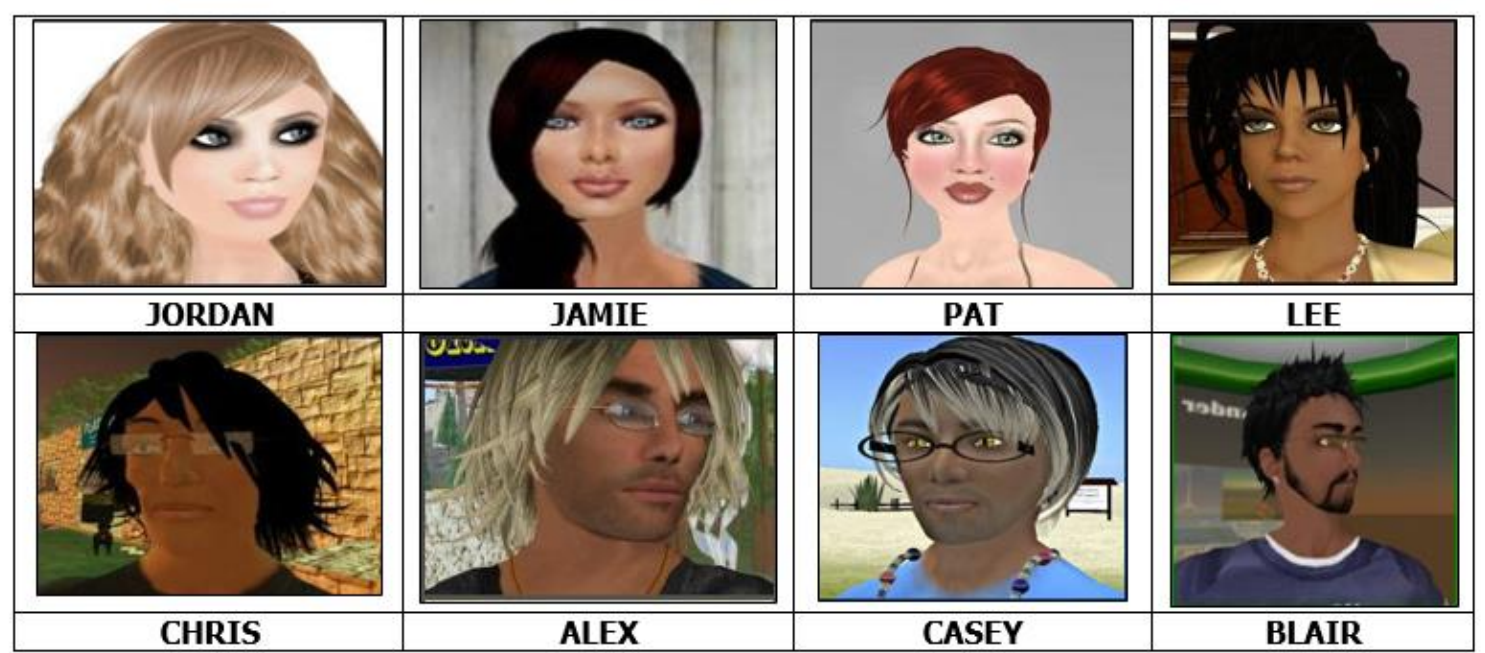

$$
\text { o Jordan o Jamie o Pat o Lee o Chris o Alex o Casey o Blair }
$$

Q1: This person "plays lacrosse for a co-ed recreational team".

Q2: This person is "an engineer and builds wind turbines and solar energy systems".

Q3: This person "teaches engineering at University of Massachusetts and also works for NASA".

Q4: This person "an airline pilot and hiked in the Appalachians last summer for 3 months".

Q5: This person is an "amateur photographer and painter".

Q6: This person "spent a year in Africa with the Peace Corps"

Q7: This person "works in the New York Stock Exchange Market".

Q8: This person "likes parachuting".

Q9: This person is a "professional actor".

Q10: This person "likes archeology and spends time in Greece".

Q11: This person "drives a hybrid car and is a make-up artist".

Q12: This person is "from Chile and likes to try different coffee flavors".

Notes: (1) for this large team condition, the eight items for the measurement of recall of team members' information consisted of items Q9-Q12 and then four random items from Q1-Q8; (2) the above figure was provided for each of the eight items, with the text of the particular item included above the images of the team members; (3) these items appeared on the post-experiment questionnaire, along with items shown in Table 6; (4) incorrect answers are recorded as zero and correct answers are recorded as one; the sum of the correct answers obtained determines the participant's recall score. 


\begin{tabular}{|l|}
\multicolumn{1}{|c|}{ Table 6. Remaining Items on the Post-Experiment Questionnaire for Condition \#5 } \\
\hline Manipulation Checks (7-point Likert scale with endpoints “Strongly Agree/Disagree"). \\
\hline Q1: Trying to remember all the descriptions about team members was overwhelming. \\
\hline Q2: I felt like there was a lot of information about the team members. \\
\hline Q3: The "Virus Outbreak" questions made me forget some of team members' descriptions. \\
\hline Attractiveness of the Visual Artifact (7-point Likert scale with endpoints "Strongly Agree/Disagree"). \\
\hline Q1: Please rate the overall attractiveness of the team members' pictures. \\
\hline $\begin{array}{l}\text { Q2: Please rate the attractiveness of the following team member's picture: (note: this item provided for each team } \\
\text { member picture) }\end{array}$ \\
\hline Interest in Interruption Task (7-point Likert scale with endpoints "Strongly Agree/ Disagree"). \\
\hline Q1: I found the "Virus Outbreak" article interesting. \\
\hline Q2: I enjoyed reading the "Virus Outbreak" article. \\
\hline Demographics \\
\hline Please indicate your age. \\
\hline Please indicate your gender (male/female). \\
\hline
\end{tabular}

The third condition presented in this paper is one of the control groups (i.e., no interruption email): small team size, with no visual artifact - condition \#9 in Table 1. This experimental condition entails background information about the experiment, which is shown in Figure 2; the introduction of four team members with no visual artifacts, which is shown in Figure 8. Subsequent is the post-experiment questionnaire, and the items that comprise this questionnaire for condition \#9 are shown in Tables 7 and 8. 


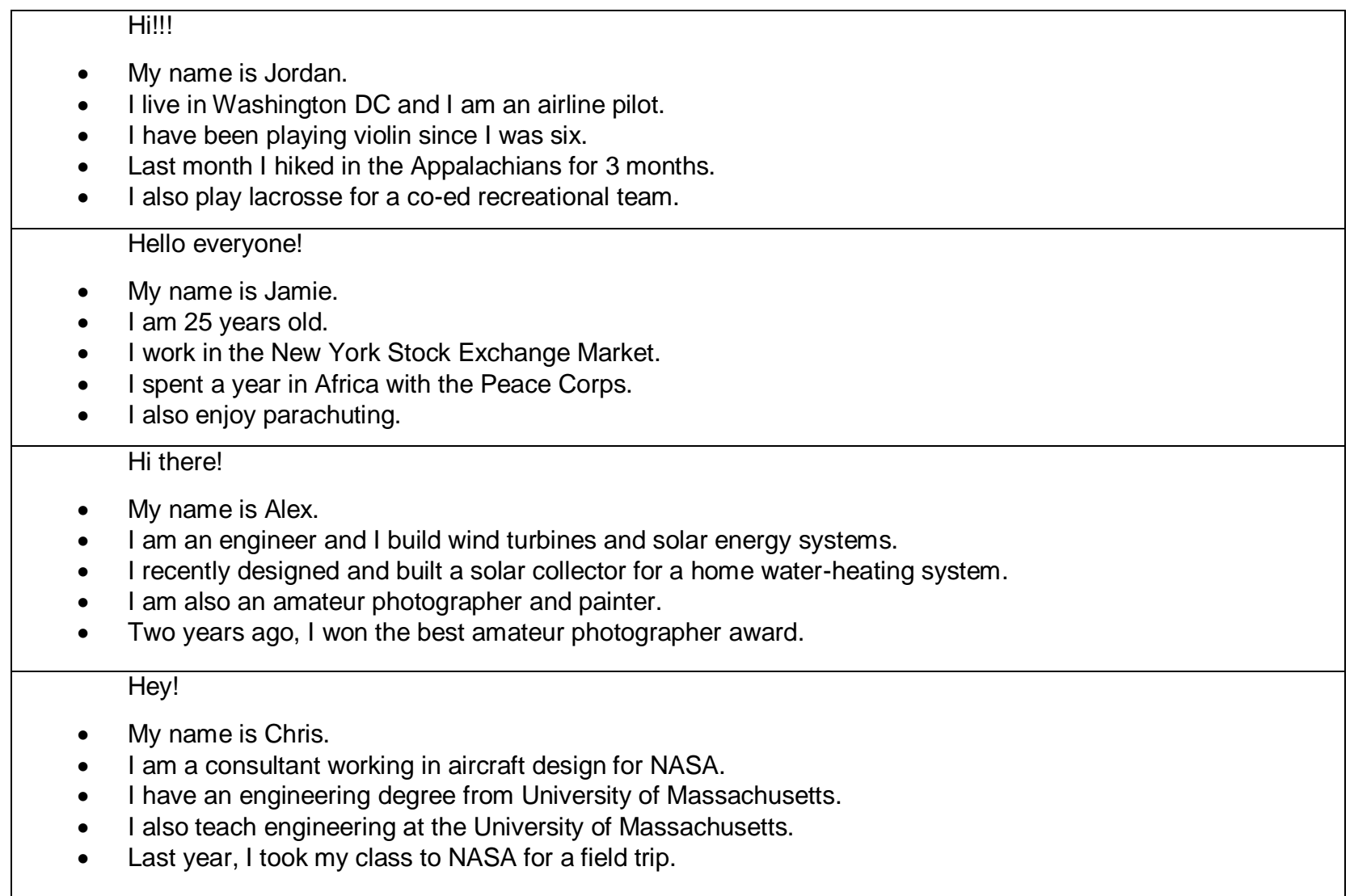

Figure 8. Team Member Information for Small Team with No Visual Artifacts (Each Displayed on a Single Webpage)

\section{Table 7. Items for Recall of Team Members' Information - Condition \#9}

Please read the description in quotes and select the team member it belongs to.

This person "spent a year in Africa with the Peace Corps".

Who is the person in the description?

$$
\text { o Jordan o Jamie o Chris o Alex }
$$

Q1: This person "plays lacrosse for a co-ed recreational team".

Q2: This person is "an engineer and builds wind turbines and solar energy systems".

Q3: This person "teaches engineering at University of Massachusetts and also works for NASA".

Q4: This person "an airline pilot and hiked in the Appalachians last summer for 3 months".

Q5: This person is an "amateur photographer and painter".

Q6: This person "spent a year in Africa with the Peace Corps"

Q7: This person "works in the New York Stock Exchange Market".

Q8: This person "likes parachuting".

Notes: (1) the above format was provided for each of the eight items; (2) these items appeared on the postexperiment questionnaire, along with items shown in Table 8 below; (3) incorrect answers are recorded as zero and correct answers are recorded as one; the sum of the correct answers obtained determines the participant's recall score. 
Table 8. Remaining Items on the Post-Experiment Questionnaire for Condition \#9

Manipulation Checks (7-point Likert scale with endpoints "Strongly Agree/Disagree").

Q1: Trying to remember all the descriptions about team members was overwhelming.

Q2: I felt like there was a lot of information about the team members.

Demographics

Please indicate your age.

Please indicate your gender (male/female).

\section{References}

Basoglu, K.A., Fuller, M.A., and Valacich, J.S. (2012). Enhancement of recall within technology-mediated teams through the use of online artifacts. ACM Transactions on Management Information Systems, 3(1), pp. 1-22. 


\section{About the Authors}

K. Asli Basoglu is an associate professor of Management Information Systems and Accounting in the Lerner College of Business at the University of Delaware. She holds a Ph.D. from Washington State University, an M.A. from the University of Virginia, and a B.A. from Bilkent University. Her research focuses mainly on decision making and performance in technology mediated work environments, and virtual teamwork. Her research has appeared in journals such as Management Information Systems Quarterly, Group Decision and Negotiations, ACM Transactions on MIS, and Accounting, Organizations, and Society.

Mark A. Fuller is Dean and Thomas O'Brien Endowed Chair at the Isenberg School of Management. He received his M.S. in Management and Ph.D. in Management Information Systems from the University of Arizona's Eller College of Management. His research focuses on virtual teamwork, technology supported learning, and trust and efficacy in technology-mediated environments, and has appeared in outlets such as Information Systems Research, Management Information Systems Quarterly, Journal of Management Information Systems, Decision Sciences, Journal of the Association for Information Systems, IEEE Transactions on Engineering Management, Journal of Organizational Behavior, and Decision Support Systems. From 2006 to 2010, he was ranked 13th worldwide in research productivity in the top four Information Systems journals. He also authored a textbook on Information Systems Project Management.

Joseph (Joe) Valacich is the Eller Professor of Management Information Systems within the Eller College of Management at the University of Arizona, a Fellow of the Association for Information Systems (2009), and the Chief Science Officer (CSO) of Neuro-ID, Inc. Dr. Valacich is a prolific scholar, publishing more than 100 scholarly articles in numerous prestigious journals. His work has had a tremendous impact on several disciplines, including information systems, computer science, psychology, marketing, and management. In December 2017, Google Scholar lists his citation counts over 21,500, with an H-index of 66. He has several pending patents related to identifying emotional state changes and indecision using human-computer interaction data. He has also coauthored several leading textbooks.

Copyright $@ 2018$ by the Association for Information Systems. Permission to make digital or hard copies of all or part of this work for personal or classroom use is granted without fee provided that copies are not made or distributed for profit or commercial advantage and that copies bear this notice and full citation on the first page. Copyright for components of this work owned by others than the Association for Information Systems must be honored. Abstracting with credit is permitted. To copy otherwise, to republish, to post on servers, or to redistribute to lists requires prior specific permission and/or fee. Request permission to publish from: AIS Administrative Office, P.O. Box 2712 Atlanta, GA, 30301-2712 Attn: Reprints or via e-mail from ais@aisnet.org. 